\title{
Ramucirumab in combination with dacarbazine in patients with progressive well-differentiated metastatic pancreatic neuroendocrine tumors (RamuNET): study protocol for a multicenter single-arm trial
}

Sebastian Krug ${ }^{1}$, Thomas Kegel ${ }^{2}$, Thomas M. Gress ${ }^{3}$, Anja Rinke ${ }^{3}$, Leonidas Apostolidis ${ }^{4}$, Henning Jann ${ }^{5}$, Alexander König ${ }^{6}$, Dieter Hörsch ${ }^{7}$ Jörg Schrader ${ }^{8}$, Thomas J. Ettrich ${ }^{9}$, Michael Richter ${ }^{10}$, Jörg Steighardt ${ }^{10}$ and Patrick Michl ${ }^{*}$

\begin{abstract}
Background: Cytotoxic chemotherapy combinations and targeted agents represent established treatment concepts in advanced pancreatic neuroendocrine tumors (PNETs). However, response rates, side effects and outcome data strongly vary among these therapeutic approaches. Head-to-head comparisons between chemo- and molecular therapies are missing and secondary resistances frequently occur. The RamuNET trial aims to identify the effectiveness of dual treatment with DTIC and ramucirumab in progressive advanced PNET patients.

Methods: The RamuNET study is an investigator-initiated multicenter prospective single-arm trial to evaluate the efficacy of ramucirumab in combination with dacarbazine (DTIC) over a period of at least 6 months. Patients with progressive well-differentiated and metastatic pancreatic neuroendocrine tumors are eligible. The study aims to include 45 patients over a period of 24 months with a minimum follow-up of 24 months. The primary endpoint is disease control after 6 months. Secondary endpoints include progression-free survival, biochemical response, overall survival, quality of life and toxicity. Based on the hypothesis that $80 \%$ of the patients can achieve a disease control after 6 months, the sample size calculation follows an exact binomial single-stage design. $\mathrm{HO}: \mathrm{p}<=\mathrm{p}_{0}=60 \%$ versus $\mathrm{H} 1: \mathrm{p}>=\mathrm{p}_{1}=80 \%$, alpha $=0.05$, beta $=0.1$.
\end{abstract}

Discussion: This study investigates a new therapeutic approach using the combination of cytotoxic and targeted antiangiogenic therapy in advanced PNET. If positive, this trial will be the basis for a randomized two-arm study to investigate the combination of ramucirumab and DTIC against other established therapies in PNET.

Trial registration: EudraCT: 2017-001207-68. Date of registration: 2018.01.03.

Keywords: PanNET, PNET, Chemotherapy, Ramucirumab, DTIC, Neuroendocrine

\footnotetext{
* Correspondence: patrick.michl@uk-halle.de

'Department of Internal Medicine I, Martin-Luther University Halle/ Wittenberg, Ernst-Grube-Str. 40, 06120 Halle (Saale), Germany

Full list of author information is available at the end of the article
}

(c) The Author(s). 2021, corrected publication 2022. Open Access This article is licensed under a Creative Commons Attribution 4.0 International License, which permits use, sharing, adaptation, distribution and reproduction in any medium or format, as long as you give appropriate credit to the original author(s) and the source, provide a link to the Creative Commons licence, and indicate if changes were made. The images or other third party material in this article are included in the article's Creative Commons licence, unless indicated otherwise in a credit line to the material. If material is not included in the article's Creative Commons licence and your intended use is not permitted by statutory regulation or exceeds the permitted use, you will need to obtain permission directly from the copyright holder. To view a copy of this licence, visit http://creativecommons.org/ licenses/by/4.0/. The Creative Commons Public Domain Dedication waiver (http://creativecommons.org/publicdomain/zero/1. 0/) applies to the data made available in this article, unless otherwise stated in a credit line to the data. 


\section{Background}

Worldwide, the incidence of neuroendocrine neoplasms (NEN) has increased over the last decades [1-3]. In contrast to metastatic NEN of the small intestine which are associated with median 10-year survival rates of $60-70 \%$, metastatic pancreatic neuroendocrine tumors (PNET) have a significantly poorer outcome $[3,4]$. Numerous efforts have been taken to improve the long-term outcome of PNET patients. However, the impact of current systemic therapeutic approaches is only modest. Recently, two treatment modalities with targeted agents have been approved: The mTOR inhibitor everolimus and the antiangiogenic multikinase inhibitor sunitinib. Both have demonstrated significant clinical efficacy in prolonging PFS in patients with pancreatic NET $[5,6]$.

Angiogenesis is a key hallmark of neuroendocrine tumors (NET). VEGF signaling has been described as major determinant of the high vascularity seen in NET both in preclinical models and in human disease [7]. Intratumoral and circulating VEGF levels have been associated with increased tumor aggressiveness and reduced survival of NET patients. Several preclinical studies and clinical trials have evaluated the impact of antiangiogenic approaches in patients with pancreatic NET [8]. The anti-angiogenic multikinase inhibitor sunitinib has shown significant effects on PFS as single agent. However, development of secondary resistance is almost inevitable. Likewise, chemotherapy with temozolomide or capecitabine in combination with the antiVEGF antibody bevacizumab showed moderate improvements of progression-free survival (PFS) in pancreatic NET (PNET) in phase II trials, but secondary resistance is common and phase III data are still missing $[9,10]$.

The anti-VEGFR2 antibody ramucirumab alone or in combination with chemotherapy has shown significant effects as second-line treatment in gastric cancer patients $[11,12]$. In contrast, antiangiogenic strategies using bevacizumab targeting VEGF as ligand have failed [13]. Similar to gastric cancer, pancreatic neuroendocrine neoplasms are characterized by high vascularity and a high stromal content containing various cellular components with high VEGF R2 expression such as macrophages and endothelial cells [14]. Based on the different VEGFR2-targeting mode of action of ramucirumab compared to VEGFtargeting bevacizumab, we hypothesized that ramucirumab is particularly effective in neuroendocrine neoplasms. Besides its efficacy as single agent and in combination with taxane-based chemotherapy in gastric cancer [11, 12], ramucirumab has been approved for non-small cell lung cancer [15] and in combination with FOLFIRI (leucovorin, fluorouracil, irinotecan) for treatment of patients with progressive metastatic colorectal cancer [16].
In NEN, streptozocin-based (STZ) chemotherapy is frequently used and recommended in symptomatic patients with high tumor load [17]. The use of the combination of doxorubicin with STZ is limited by potential cumulative cardiotoxicity (maximum doxorubicin dose must be less than $500 \mathrm{mg} / \mathrm{m}^{2}$ ) and has been largely replaced by the use of the combination of 5-fluorouracil (5-FU) with STZ [18].

However, randomized phase III data are lacking and thus most evidence was achieved with recently published large retrospective studies [19-21]. An alternative therapeutic option is the alkylating drug temozolomide (TEM) or its derivative dacarbazine (DTIC). While TEM is routinely combined with capecitabine, thus presenting an attractive orally available doublet [9], DTIC monotherapy was recently reinvigorated, based on a large retrospective evaluation [22]. Here, DTIC at a dose of $650 \mathrm{mg} / \mathrm{m}^{2}$ was used. A 4-week schedule and a favourable toxicity profile represent relevant advantages of DTIC.

\section{Rationale for the trial}

VEGF receptor-2 (VEGFR2) is the premier receptor responsible for many of the cancer phenotypes, including modification of blood vessel structure and function, proliferation and migration [7, 23]. The fully human monoclonal antibody ramucirumab specifically and potently inhibits VEGF receptor-2 [24, 25].

Based on this specificity, ramucirumab has potential advantages over most other antiangiogenic drugs such as bevacizumab as it is selective for VEGFR2, whereas bevacizumab by targeting VEGF-A affects VEGFR1, -R2, and the noncatalytic coreceptors neuropilin-1and -2 . Ramucirumab thus leaves the VEGFR1 receptor which functions as decoy receptor unaffected, thereby further enhancing the VEGFR2 inhibitory effect [24, 25]. Preclinical and clinical data indicate a pivotal role of VEGF R2 in promoting tumor vasculature and progression in PNETs and suggest an impact of VEGFR2 on acquired resistance [14, 26-28].

In addition, VEGFR2 is highly expressed on macrophages, rendering them a relevant target of ramucirumab. Inhibition of VEGFR2 on macrophages results in decreased tumor immune infiltration, cytokine and chemokine release, leading to impaired tumor growth and proliferation [29, 30]. Own preclinical data indicate that macrophages are expressed at high levels in human and murine pancreatic neuroendocrine tumor tissues and are predominantly polarized towards a protumoral, proangiogenic M2 phenotype, therefore representing a promising target for therapeutic intervention.

Combination regimens using a DTIC-based chemotherapeutic backbone combined with ramucirumab may inhibit early therapy-induced resistance mechanisms by 
targeting angiogenesis synergistically through different mechanisms. Several preclinical or clinical reports demonstrated a marked antiangiogenic action of DTIC or its oral derivative temozolomide indicating synergism with antiangiogenic targeted agents [10,31].

Moreover, the combination of ramucirumab and DTIC has already been demonstrated as feasible and associated with an acceptable safety profile in a randomized phase II trial in patients with malignant melanoma [10, 31]. Furthermore, ramucirumab was studied in patients with pretreated gastric neuroendocrine carcinomas in combination with chemotherapy. The results were promising and demonstrated a benefit of dual therapy as opposed to chemotherapy alone [32].

Based on the unique molecular mode of action of ramucirumab and the synergistic potential of DTIC, the combination therapy with both agents represents a promising strategy for progressive pancreatic neuroendocrine neoplasms.

\section{Methods}

Study endpoints

Primary endpoints

Primary endpoint is the disease-control (DC) at 6 months as assessed by RECIST 1.1 criteria. Diseasecontrol will be calculated from start of study treatment until progressive disease. DC is the most important parameter to assess the efficacy of a novel therapy regimen in the population of patients with PNET who frequently undergo multiple lines of loco-regional and systemic treatments.

If a positive DC signal with the combination of ramucirumab and DTIC is observed in this pilot trial, a subsequent evaluation in a randomized phase II/III trial is justified.

\section{Secondary endpoints}

- Objective tumor response (OR)

- progression-free survival (PFS)

- overall survival (OS)

- toxicities

- biochemical response (tumor marker chromogranin A (CgA); in cases of functional pancreatic NET: the specific hormone can be evaluated

- QoL (EORTC QLQ-C30 questionnaire)

\section{Study population}

Patients with histologically confirmed unresectable metastatic non-functional or functional G1-G2 PNET excluding G3 neuroendocrine carcinomas (NEC) or G3 neuroendocrine tumors (NET), who have progressive disease under treatment with either non-DTIC-based chemotherapy (e.g. 5-FU/ streptozocin, capecitabine),
SSA analogues, everolimus, sunitinib or PRRT are eligible for screening and enrollment in the trial. The Investigator will keep a record of all study candidates who were considered for enrollment including screening failures.

\section{Inclusion criteria}

- Histologically confirmed unresectable metastatic non-functional or functional G1-G2 PNET excluding G3 neuroendocrine carcinomas (NEC)

- Age: $18-75$ years

- Measurable disease (RECIST 1.1)

- Progressive disease under treatment with either non-DTIC-based chemotherapy (e.g. 5-FU/streptozocin, capecitabine), SSA analogues, everolimus, sunitinib or PRRT. No prior therapy with DTIC is allowed.

- ECOG 0-1

- Life expectancy $>12$ weeks

- Adequate renal function (serum creatinine $\leq 1.5 \mathrm{x}$ ULN, or creatinine clearance (measured via 24-h urine collection) $\geq 40 \mathrm{~mL} /$ minute (if serum creatinine is $>1.5 \mathrm{x}$ ULN, a $24-\mathrm{h}$ urine collection to calculate creatinine clearance must be per-formed). Urinary protein is $\leq 1+$ on dipstick or routine urinalysis (UA; if urine dipstick or routine analysis is $\geq 2+$, a $24-\mathrm{h}$ urine collection for protein must demonstrate < $1000 \mathrm{mg}$ of protein in $24 \mathrm{~h}$ to allow participation in this protocol).

- Adequate hepatic function (total bilirubin $\leq 1.5$ $\mathrm{mg} / \mathrm{dL}(25.65 \mu \mathrm{mol} / \mathrm{L})$, and aspartate transaminase (AST) and alanine transaminase (ALT) $\leq 3.0 \mathrm{x}$ ULN; or $5.0 \times$ ULN in the setting of liver metastases)

- Adequate bone marrow function (absolute neutrophil count $>1500 / \mathrm{mm}^{3}$, platelets $>100,000 /$ $\mathrm{mm}^{3}$, hemoglobin $>9 \mathrm{~g} / \mathrm{dL}$ )

- Adequate coagulation function (INR $\leq 1.5$ and $\mathrm{PTT} \leq 5 \mathrm{~s}$ above the ULN (unless receiving anticoagulation therapy)

- Pathological condition present that carries a high risk of bleeding (for example, tumor involving major vessels or known varices)

- The patient, if sexually active, must be postmenopausal, surgically sterile, or using effective contraception (hormonal or barrier methods, Pearl Index $<1$ )

- Female patients of childbearing potential must have a negative serum pregnancy test within 7 days prior to first dose of protocol therapy

- The patient must be able to understand, consent and sign the written consent form 


\section{Exclusion criteria}

- Pregnancy (positive urine or blood pregnancy test) or lactation.

- Secondary malignancies in patient's history with the exception of: disease-free period $>5$ years before randomization or basalioma of the skin or carcinoma of the cervix after successful therapy

- Allergy against dacarbacine or ramucirumab

- Current enrolment or participation within the last 4 weeks in a clinical drug trial

- Any arterial thromboembolic events, including but not limited to myocardial infarction, transient ischemic attack, cerebrovascular accident, or unstable angina, within 6 months prior to first dose of protocol therapy

- Insufficient liver function: cirrhosis at a level of Child-Pugh B (or worse) or cirrhosis (any degree) and a history of hepatic encephalopathy or clinically meaningful ascites resulting from cirrhosis. Clinically meaningful ascites is defined as ascites from cirrhosis requiring diuretics or paracentesis.

- Uncontrolled or poorly-controlled hypertension (> $160 \mathrm{mmHg}$ systolic or $>100 \mathrm{mmHg}$ diastolic for $>4$ weeks) despite standard medical management

- Chronic antiplatelet therapy, including aspirin, nonsteroidal anti-inflammatory drugs (NSAIDs, including ibuprofen, naproxen, and others), dipyridamole or clopidogrel, or similar agents. Once-daily aspirin use (maximum dose $325 \mathrm{mg} /$ day) is permitted

- Grade 3-4 GI bleeding within 3 months prior to first dose of protocol therapy.

- History of deep vein thrombosis (DVT), pulmonary embolism (PE), or any other significant thromboembolism (venous port or catheter thrombosis or superficial venous thrombosis are not considered "significant") during the 3 months prior to first dose of protocol therapy

- Uncontrolled severe physical or mental disorders such as: neurological or psychiatric disorders including seizure, advanced dementia, psychosis, active uncontrolled infections or sepsis, HIV, replicative hepatitis $\mathrm{B}$ or $\mathrm{C}$ infection

- Pathological condition present that carries a high risk of bleeding (for example, tumor involving major vessels or known varices)

- History of gastrointestinal perforation/fistula (within 6 months of first dose of protocol therapy) or risk factors for perforation.

- Major surgery within 28 days prior to first dose of protocol therapy, or minor sur-gery/subcutaneous venous access device placement within 7 days prior to first dose of protocol therapy
- Elective or planned major surgery to be performed during the course of the clinical trial.

- Serious or nonhealing wound, ulcer, or bone fracture within 28 days prior to first dose of protocol therapy.

\section{Trial design}

RamuNET is a multicenter single-arm pilot study. Patients are recruited in approximately 8 participating centres that are all University hospitals or tertiary referral hospitals in Germany with excellent expertise in the care of patients with neuroendocrine neoplasms. A recruitment of an average of 5 patients with progressive PNET per centre over a period of 12 months seems realistic.

\section{Conduct of the trial}

There is a screening period of 28 days. All required study procedures are listed in Table 1. After study enrollment, therapy continues for 6 months (primary endpoint) or until tumor progression. If patients are stable after month 6, treatment can be continued until progression or toxicity). Patients will be followed-up at least 24 months from the start of the study. Finally, the final data analysis is performed (Fig. 1).

\section{Study treatment}

Ramucirumab at a dose of $8 \mathrm{mg} / \mathrm{kg}$ body weight, is administered i.v. on days 1 and 15 of each 28-day cycle, over a time period of $60 \mathrm{~min}$ prior to administration of dacarbazine (DTIC). DTIC is given at a dose of $650 \mathrm{mg} /$ $\mathrm{m}^{2}$ body surface area on day 1 and then once every 4 weeks as intravenous infusion over $30 \mathrm{~min}$ as previously described [22]. Overall the study treatment includes 6 cycles of the combination therapy (Fig. 2).

\section{Translational program}

This study concept is supported by own preclinical data indicating high amounts of VEGFR2 positive tumorinfiltrating macrophages in PNET tissues as therapeutic targets as well as literature reports from other groups and clinical trials in other tumor entities supporting the combination of anti-VEGFR2 together chemotherapy including DTIC. These data indicate that systemic administration of ramucirumab may overcome therapy resistance by affecting both the tumor cell compartment and also the local and systemic innate immune response, leading to a modulation of tumor-associated macrophages towards an antimetastatic phenotype.

The protocol includes analysis of biopsy specimens as well as resected tumor specimens in case of previous surgeries in the participating patients. The aim of the translational program is to correlate response patterns to ramucirumab with characteristic features in the tumor tissue. All participating centers are requested to 
Table 1 Requirements for the study

\begin{tabular}{|c|c|c|c|c|c|c|c|}
\hline \multirow{2}{*}{$\begin{array}{l}\text { Study Procedures } \\
\text { Study Day }\end{array}$} & \multirow{2}{*}{$\begin{array}{c}\begin{array}{c}\text { Screen- } \\
\text { ing }\end{array} \\
\text { Day -14 to } \\
\text { Day } 0\end{array}$} & \multicolumn{2}{|c|}{$\begin{array}{c}\text { Cycle } 1,2,3,5,6 ; \\
\text { continiued } \\
\text { until PD }\end{array}$} & \multicolumn{2}{|c|}{$\begin{array}{l}\text { Cycle } 4 \text { and every } \\
\text { following } 3^{\text {rd }} \text { cycle }\end{array}$} & \multirow[t]{2}{*}{ EOT } & \multirow{2}{*}{$\begin{array}{c}\begin{array}{c}\text { Follow up } \\
\text { after EOT }\end{array} \\
\text { every } 3 \\
\text { months } \\
\pm 7 \text { days }\end{array}$} \\
\hline & & Day 1 & $\begin{array}{l}\text { Day } 15 \\
\quad \pm 2\end{array}$ & $\begin{array}{l}\text { Day } 1 \\
\pm 2\end{array}$ & $\begin{array}{l}\text { Day } 15 \\
\quad \pm 2\end{array}$ & & \\
\hline Informed consent & $\mathrm{X}$ & & & & & & \\
\hline Inclusion/exclusion criteria & $x$ & & & & & & \\
\hline Physical examination & & $x$ & & $x$ & & $\mathrm{X}$ & \\
\hline Demographic \& medical history ${ }^{1}$ & $\mathrm{X}$ & & & & & & \\
\hline $\begin{array}{l}\text { Body weight, height (only at } \\
\text { screening) }\end{array}$ & $x$ & $x$ & $\mathrm{x}$ & $x$ & $x$ & $\mathrm{x}$ & \\
\hline Vital signs (RR, Puls) & $x$ & $\mathrm{x}$ & $\mathrm{x}$ & $x$ & $\mathrm{x}$ & & \\
\hline ECOG & $x$ & $x$ & $x$ & $x$ & $x$ & & \\
\hline Quality of Life (EORTC QLQ-C30) & $\mathrm{x}$ & & & $\mathrm{x}$ & & $x$ & \\
\hline Complete Blood Count & $\mathrm{x}$ & $x$ & $x$ & $x$ & $\mathrm{X}$ & $\mathrm{X}$ & \\
\hline Differential Blood Count & $\mathrm{x}$ & $\mathrm{x}$ & $\mathrm{x}$ & $\mathrm{x}$ & $\mathrm{x}$ & $\mathrm{x}$ & \\
\hline Biochemistry & $\mathrm{X}$ & $\mathrm{X}$ & & $\mathrm{x}$ & & $\mathrm{X}$ & \\
\hline Coagulation & $\mathrm{X}$ & & & & & & \\
\hline $24 \mathrm{~h}$ urine analysis & & $\mathrm{x}$ & & $\mathrm{x}$ & & $\mathrm{x}$ & \\
\hline Tumor markers & $\mathrm{X}$ & & & $\mathrm{X}$ & & $\mathrm{x}$ & \\
\hline Pregnancy test & $\mathrm{X}$ & $\mathrm{X}$ & & $\mathrm{X}^{3}$ & & & \\
\hline ECG & $\mathrm{X}$ & & & & & & \\
\hline CT / MRI of thorax and abdomen & $\mathrm{X}$ & & & $x^{1}$ & & $\mathrm{x}^{2}$ & \\
\hline $\begin{array}{l}\text { Tumor tissue acquisition (rebiopsy } \\
\text { if older than } 6 \text { months) }\end{array}$ & $\mathrm{x}$ & & & & & & \\
\hline Serum collection for biomarkers & $\mathrm{x}$ & & & $\mathrm{x}$ & & $\mathrm{X}$ & \\
\hline Overall survival status by phone & & & & & & & $\mathrm{x}$ \\
\hline Ramicirumab i.v. injections & & $x$ & $x$ & $x$ & $x$ & & \\
\hline Dacarbazine Chemotherapy & & $x$ & & $x$ & & & \\
\hline Adverse events & $\longleftarrow$ & & & & & & $x^{4}$ \\
\hline Concomitant medications & & & & & & & \\
\hline
\end{tabular}

1every 3 months;

${ }^{2}$ if no imaging has been performed within the last 4 weeks.

${ }^{3}$ to be performed at the beginning of each cycle.

${ }^{4}$ to be followed beyond the 30 days until resolution or stabilization

send tissue blocks from biopsies or previous surgeries for central translational analyses.

Histopathological analysis of tumor specimens will be performed centrally at the Institute of Pathology, Martin Luther University of Halle, Germany. This includes confirmatory analysis of proliferation rate (Ki-67) and tumor grading as well as VEGFR2 expression in tumor and surrounding stromal cells. In addition, infiltration of macrophages (CD68, CD204, CD163, VEGFR2) and different T cell subpopulations (CD3/CD8: cytotoxic T cells, FoxP3: regulatory $\mathrm{T}$ cells) will be assessed to correlate infiltration with tumor-associated macrophages and cytotoxic or regulatory $\mathrm{T}$ cells with subsequent response to ramucirumab therapy. In addition, immunohistochemical analyses will be performed for angiogenesis (microvessel density; CD31) and epithelial-mesenchymal transition (EMT, E-cadherin, vimentin). Both angiogenesis and EMT are known to be modulated by tumor-associated
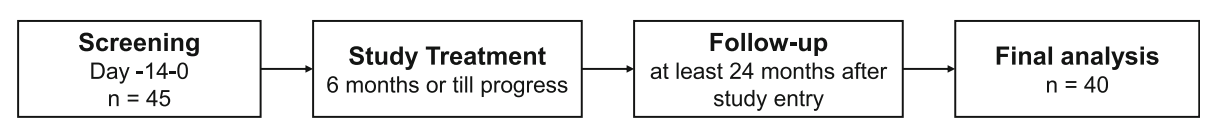

Fig. 1 Study procedure 


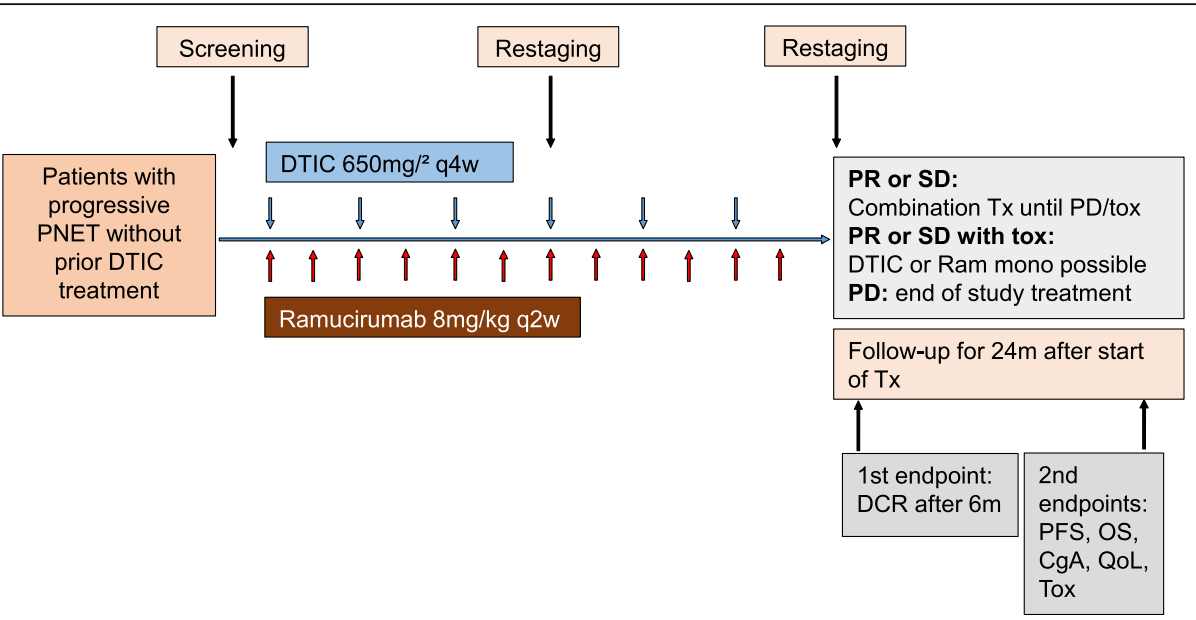

Fig. 2 Therapy schedule and endpoints of the study

macrophages and modulate metastasis formation and might serve as predictors of response to bisphosphonates.

In addition to immunohistochemical analyses on resected tumor specimens, we will examine circulating biomarkers in serum to identify predictors of response to ramucirumab or prognostic parameters as liquid biopsies. Serum and plasma will be used for central analysis of circulating, macrophage-associated cytokines and mediators of angiogenesis (e.g. IL1, IL6, VEGF, Angiopoetin-1 / -2) and correlated to clinical parameters.

\section{Statistical analyses}

This trial is planned as a pilot study to evaluate the efficacy of combination treatment of ramucirumab and dacarbazine. Based on the hypothesis that $80 \%$ of the patients can achieve a disease control $(\mathrm{DCR}=\mathrm{CR}, \mathrm{PR}$ and SD) after 6 months. The sample size calculation follows an exact binomial single-stage design [33]. $\mathrm{H} 0: \mathrm{p}<=\mathrm{p}_{0}=$ $60 \%$ versus $H 1: p>=p_{1}=80 \%$, alpha $=0.05$, beta $=0.1$. 45 patients will be recruited in the participating centers during a period of one year. If the primary endpoint of this pilot trial lies within in the confidence interval, thereby demonstrating clinical efficacy of the combination treatment, the aim is to corroborate these data in a randomized phase II study.

\section{Patient and public involvement}

The patient organization Netzwerk Neuroendokrine Tumoren (NeT) e.V. (https://www.netzwerk-net.de; German patient support group for patients with neuroendocrine tumor diseases) was involved and provided precious advice during the planning of the trial and the construction of the trial protocol. Upon trial completion and availability of results, patient involvement will be sought to disseminate the results within the patient community and the public. Furthermore, the present study procotol was presented and discussed in regional and national patient events of the participating centers.

\section{Ethical considerations}

The study will be conducted in compliance with Good Clinical practice (GCP) and the applicable national laws and regulations to assure that the rights, safety, and well-being of the participating subjects are protected consistent with the ethical principles that have their origin in the Declaration of Helsinki. Protocol amendments will be submitted to the ethics committee and signed by all authors of the trial protocol. The Clinical Trials Centre Halle (KKS) is an external party that will monitor the study in a risk-adapted way and ensure that the study follows GCP, that is, that all participants give informed written consent.

\section{Data management}

All data will be captured on paper-based case report forms at the trial site (Case Report Forms - CRF) and transferred to the data management of the KKS Halle. The study management software secuTrial ${ }^{\oplus}$, a GCP compliant clinical data management system for the conduction of clinical trials will be used for data entry and query management. All changes in the data will be recorded by an audit trail. The study software provides an adaptive concept for user accounts and user roles depending on the study. The data base is integrated in a general IT infrastructure and security concept including a firewall and backup system. The data will be saved on a daily basis. Data will be frequently captured into a data base at KKS Halle. After completion of data capture and quality check the data base will be closed and data will be transferred to the biometrician for statistical analysis. 


\section{Dissemination strategy}

Findings of this study will be disseminated to participants, healthcare professionals, the public and other relevant groups. The results of this study will be published open access in a peer-reviewed scientific journal and presented at national and international conferences. The principal investigator will review all manuscripts. The authorship list will be agreed on by the principal investigator prior to publication. Publication of the first manuscript reporting study results is planned after analysis of the primary endpoint.

\section{Discussion}

Chemotherapy with streptozocin (STZ) and 5-FU is considered as first-line treatment in patients with progressive well-differentiated pancreatic neuroendocrine tumor (PNET), although the studies were published a considerable time ago [18, 34]. Recently, somatostatin analogues were shown to prolong the progression-free survival in a subgroup of non-functional PNET patients. After failure of first-line treatment or severe toxicities targeted molecules such as everolimus and sunitinib have been approved after phase III trials demonstrated improvement of PFS. Besides these two trials, randomized therapeutic studies in PNET are rare, given the low prevalence of this disease. Watch and wait strategies in advanced unresectable PNET patients are no longer feasible based on the CLARINET trial demonstrating a benefit for the somatostatin analogue (SSA) lanreotide in PNET. Among all neuroendocrine tumors, the pancreatic tumor site and stage IV disease represent negative prognostic factors. In contrast to low-proliferating NET tumors in other locations for which watch-and-wait strategies can be justified, almost all advanced PNET patients are eligible to receive treatment.

In addition to the fact that randomized evidence on the available therapeutic options is limited, the optimal sequence of available therapeutic options remains also controversial. Since PNET patients frequently undergo multiple lines of treatment and median overall survival (OS) ranges between 5 and 10 years, the influence of one particular therapeutic regimen and its timing in the sequence of therapy lines on OS is moderate hard to assess. Additionally, the combination of different therapeutic protocols e.g. SSA in combination with chemotherapy or targeted agents is not evidence-based but is frequently applied clinical routine. The evaluation of the biologically meaningful combination treatment with the cytotoxic agent dacarbazine (DTIC) and the antiangiogenic molecule ramucirumab represents a first step to generate new evidence in this context and to overcome resistance to currently available treatment options in PNET.

\section{Trial status}

Protocol version 01 was released in August 2018. Recruitment started in June 2019 and the participating centres were successively initiated. Actually, 25 patients have been recruited by all centres. The recruitment period is expected to last another 18 months (status September 2021).

\begin{abstract}
Abbreviations
CTx: chemotherapy; STZ: streptozocin; TEM: temozolomide; DTIC: dacarbazine; CAP: capecitabine; NEN: neuroendocrine neoplasms: NET: neuroendocrine tumor; w: week; d: day; PFS: progression-free survival; OS: overall survival; RR: response rate; OR: objective response; ORR: objective response rate; DC: disease control; DCR: disease control rate; CR: complete response; PR: partial response; SD: stable disease; PD: progressive disease; ULN: upper level of normal
\end{abstract}

\section{Acknowledgements}

We would like to thank the participating centres of the RamuNET trial.

\section{Authors' contributions}

SK and PM conducted the study and drafted the trial protocol. MR and JS organized the formalities and the course of the study as a clinical study centre. AR, TK, JS, LA, AK, HJ, DH, TMG and TE were involved in the recruitment of patients within their centres. All authors critically reviewed and amended the protocol. All authors read and approved the final manuscript.

\section{Funding}

The trial is funded by Eli Lilly and Company. The funding body was involved in the design of the study. Collection, analysis, and interpretation of data is performed independently. The study underwent peer-review by the funding body. The funding body was not implicated in writing the manuscript. Open Access funding enabled and organized by Projekt DEAL.

Availability of data and materials

Not applicable, no datasets are included in this study protocol.

\section{Declarations}

Ethics approval and consent to participate

This study was approved by the ethics committee of the Martin Luther University Halle/Wittenberg (protocol number: KKSH140). The local medical ethics committees of the participating centers gave positive advice on the local feasibility of the trial. The study will be conducted in accordance with the ethical principles of the Declaration of Helsinki. Informed consent will be obtained from all study participants. The RamuNET trial has been registered in the ClinicalTrials.gov registry (EudraCT: 2017-001207-68).

\section{Consent for publication}

Not applicable.

\section{Competing interests}

The authors declare that they have no competing interests.

\section{Author details}

1Department of Internal Medicine I, Martin-Luther University Halle/ Wittenberg, Ernst-Grube-Str. 40, 06120 Halle (Saale), Germany. ${ }^{2}$ Department of Internal Medicine IV, Martin-Luther University Halle/Wittenberg, Halle, Germany. ${ }^{3}$ Department of Gastroenterology, Endocrinology and Metabolism, Philipps-University Marburg, Marburg, Germany. ${ }^{4}$ Department of Medical Oncology, National Center for Tumor Diseases, University Hospital Heidelberg, Heidelberg, Germany. ${ }^{5}$ Department of Gastroenterology and Hepatology, Charité University Hospital, Berlin, Germany. ${ }^{6}$ Department of Gastroenterology and gastrointestinal Oncology, Georg-August University, Göttingen, Germany. Department of Gastroenterology/Endocrinology, Center for Neuroendocrine Tumors Bad Berka, Bad Berka, Germany. ${ }^{8}$ Department of Medicine - Gastroenterology and Hepatology, University Medical Center Hamburg-Eppendorf, Hamburg, Germany. ${ }^{9}$ Department of 
Internal Medicine I, University of UIm, UIm, Germany. ${ }^{10}$ Coordination Centre for Clinical Trials, Faculty of Medicine, Martin-Luther University Halle/ Wittenberg, Halle, Germany.

Received: 16 September 2020 Accepted: 21 October 2021

Published online: 12 November 2021

\section{References}

1. Yao JC, Hassan M, Phan A, Dagohoy C, Leary C, Mares JE, et al. One hundred years after "carcinoid": epidemiology of and prognostic factors for neuroendocrine tumors in 35,825 cases in the United States. J Clin Oncol. 2008;26(18):3063-72. https://doi.org/10.1200/JCO.2007.15.4377.

2. Lawrence B, Gustafsson BI, Chan A, Svejda B, Kidd M, Modlin IM. The epidemiology of gastroenteropancreatic neuroendocrine tumors. Endocrinol Metab Clin North Am. 2011;40(1):1-18, vii. https://doi.org/10.1016/j.ecl.201 0.12 .005 .

3. Halfdanarson TR, Rabe KG, Rubin J, Petersen GM. Pancreatic neuroendocrine tumors (PNETs): incidence, prognosis and recent trend toward improved survival. Ann Oncol. 2008;19(10):1727-33. https://doi.org/10.1093/annonc/ mdn351.

4. Pape UF, Berndt U, Müller-Nordhorn J, Böhmig M, Roll S, Koch M, et al. Prognostic factors of long-term outcome in gastroenteropancreatic neuroendocrine tumours. Endocr Relat Cancer. 2008;15(4):1083-97. https:// doi.org/10.1677/ERC-08-0017.

5. Raymond E, Dahan L, Raoul JL, Bang YJ, Borbath I, Lombard-Bohas C, et al. Sunitinib malate for the treatment of pancreatic neuroendocrine tumors. N Engl J Med. 2011;364(6):501-13. https://doi.org/10.1056/NEJMoa1003825.

6. Yao JC, Shah MH, Ito T, Bohas CL, Wolin EM, Van Cutsem E, et al. Everolimus for advanced pancreatic neuroendocrine tumors. N Engl J Med. 2011;364(6): 514-23. https://doi.org/10.1056/NEJMoa1009290.

7. Bergers G, Javaherian K, Lo KM, Folkman J, Hanahan D. Effects of angiogenesis inhibitors on multistage carcinogenesis in mice. Science. 1999; 284(5415):808-12. https://doi.org/10.1126/science.284.5415.808.

8. Scoazec JY. Angiogenesis in neuroendocrine tumors: therapeutic applications. Neuroendocrinology. 2013;97(1):45-56. https://doi.org/10.1159/ 000338371.

9. Strosberg JR, Fine RL, Choi J, Nasir A, Coppola D, Chen DT, et al. First-line chemotherapy with capecitabine and temozolomide in patients with metastatic pancreatic endocrine carcinomas. Cancer. 2011;117(2):268-75. https://doi.org/10.1002/cncr.25425.

10. Chan JA, Stuart K, Earle CC, Clark JW, Bhargava P, Miksad R, et al. Prospective study of bevacizumab plus temozolomide in patients with advanced neuroendocrine tumors. J Clin Oncol. 2012;30(24):2963-8. https://doi.org/1 $0.1200 / J C O .2011 .40 .3147$

11. Fuchs CS, Tomasek J, Yong CJ, Dumitru F, Passalacqua R, Goswami C, et al. Ramucirumab monotherapy for previously treated advanced gastric or gastro-oesophageal junction adenocarcinoma (REGARD): an international, randomised, multicentre, placebo-controlled, phase 3 trial. Lancet. 2014; 383(9911):31-9. https://doi.org/10.1016/S0140-6736(13)61719-5.

12. Wilke H, Muro K, Van Cutsem E, Oh SC, Bodoky G, Shimada Y, et al. Ramucirumab plus paclitaxel versus placebo plus paclitaxel in patients with previously treated advanced gastric or gastro-oesophageal junction adenocarcinoma (RAINBOW): a double-blind, randomised phase 3 trial. Lancet Oncol. 2014;15(11):1224-35. https://doi.org/10.1016/S1470-2045(14 )70420-6.

13. Ohtsu A, Shah MA, Van Cutsem E, Rha SY, Sawaki A, Park SR, et al. Bevacizumab in combination with chemotherapy as first-line therapy in advanced gastric cancer: a randomized, double-blind, placebo-controlled phase III study. J Clin Oncol. 2011;29(30):3968-76. https://doi.org/10.1200/ JCO.2011.36.2236.

14. Speisky D, Duces A, Bièche I, Rebours V, Hammel P, Sauvanet A, et al. Molecular profiling of pancreatic neuroendocrine tumors in sporadic and Von Hippel-Lindau patients. Clin Cancer Res. 2012;18(10):2838-49. https:// doi.org/10.1158/1078-0432.CCR-11-2759.

15. Garon EB, Ciuleanu TE, Arrieta O, Prabhash K, Syrigos KN, Goksel T, et al. Ramucirumab plus docetaxel versus placebo plus docetaxel for second-line treatment of stage IV non-small-cell lung cancer after disease progression on platinum-based therapy (REVEL): a multicentre, double-blind, randomised phase 3 trial. Lancet. 2014;384(9944):665-73. https://doi.org/10.1 016/S0140-6736(14)60845-X.
16. Tabernero J, Yoshino T, Cohn AL, Obermannova R, Bodoky G, GarciaCarbonero $\mathrm{R}$, et al. Ramucirumab versus placebo in combination with second-line FOLFIRI in patients with metastatic colorectal carcinoma that progressed during or after first-line therapy with bevacizumab, oxaliplatin, and a fluoropyrimidine (RAISE): a randomised, double-blind, multicentre, phase 3 study. Lancet Oncol. 2015;16(5):499-508. https://doi.org/10.1016/ S1470-2045(15)70127-0.

17. Pavel M, O'Toole D, Costa F, Capdevila J, Gross D, Kianmanesh R, et al. ENET $\mathrm{S}$ consensus guidelines update for the Management of Distant Metastatic Disease of intestinal, pancreatic, bronchial neuroendocrine neoplasms (NEN) and NEN of unknown primary site. Neuroendocrinology. 2016;103(2):172-85. https://doi.org/10.1159/000443167.

18. Moertel CG, Lefkopoulo M, Lipsitz S, Hahn RG, Klaassen D. Streptozocindoxorubicin, streptozocin-fluorouracil or chlorozotocin in the treatment of advanced islet-cell carcinoma. N Engl J Med. 1992;326(8):519-23. https://doi. org/10.1056/NEJM199202203260804.

19. Clewemar Antonodimitrakis $P$, Sundin A, Wassberg C, Granberg D, Skogseid B, Eriksson B. Streptozocin and 5-FU for the treatment of pancreatic neuroendocrine tumors: efficacy, Prognostic Factors and Toxicity. Neuroendocrinology. 2016;103(3-4):345-53. https://doi.org/10.1159/00043 9086. Epub 2015 Aug 7.

20. Dilz LM, Denecke T, Steffen IG, Prasad V, von Weikersthal LF, Pape UF, et al. Streptozocin/5-fluorouracil chemotherapy is associated with durable response in patients with advanced pancreatic neuroendocrine tumours. Eur J Cancer. 2015;51(10):1253-62. https://doi.org/10.1016/j.ejca.2015.04.005.

21. Krug S, Boch M, Daniel H, Nimphius W, Müller D, Michl P, et al. Streptozocin-based chemotherapy in patients with advanced neuroendocrine neoplasms - predictive and prognostic markers for treatment stratification. PLoS One. 2015;10(12):e0143822. https://doi.org/1 0.1371/journal.pone.0143822.

22. Mueller D, Krug S, Majumder M, Rinke A, Gress TM. Low dose DTIC is effective and safe in pretreated patients with well differentiated neuroendocrine tumors. BMC Cancer. 2016;16(1):645. https://doi.org/10.11 86/s12885-016-2642-1.

23. Casanovas O, Hicklin DJ, Bergers G, Hanahan D. Drug resistance by evasion of antiangiogenic targeting of VEGF signaling in late-stage pancreatic islet tumors. Cancer Cell. 2005;8(4):299-309. https://doi.org/10.1016/j.ccr.2005.09.005.

24. Spratlin JL, Cohen RB, Eadens M, Gore L, Camidge DR, Diab S, et al. Phase I pharmacologic and biologic study of ramucirumab (IMC-1121B), a fully human immunoglobulin G1 monoclonal antibody targeting the vascular endothelial growth factor receptor-2. J Clin Oncol. 2010;28(5):780-7. https:// doi.org/10.1200/JCO.2009.23.7537

25. Spratlin JL, Mulder KE, Mackey JR. Ramucirumab (IMC-1121B): a novel attack on angiogenesis. Future Oncol. 2010;6(7):1085-94. https://doi.org/10.2217/ fon.10.75.

26. Rigamonti N, Kadioglu E, Keklikoglou I, Wyser Rmili C, Leow CC, De Palma M. Role of angiopoietin-2 in adaptive tumor resistance to VEGF signaling blockade. Cell Rep. 2014;8(3):696-706. https://doi.org/10.1016/j.celrep.2014. 06.059 .

27. Allen E, Walters IB, Hanahan D. Brivanib, a dual FGFNEGF inhibitor, is active both first and second line against mouse pancreatic neuroendocrine tumors developing adaptive/evasive resistance to VEGF inhibition. Clin Cancer Res. 2011;17(16):5299-310. https://doi.org/10.1158/1078-0432.CCR-10-2847.

28. Huang YT, Lan Q, Ponsonnet L, Blanquet M, Christofori G, Zaric J, et al. The matricellular protein CYR61 interferes with normal pancreatic islets architecture and promotes pancreatic neuroendocrine tumor progression. Oncotarget. 2016;7(2):1663-74. https://doi.org/10.18632/oncotarget.6411.

29. Dineen SP, Lynn KD, Holloway SE, Miller AF, Sullivan JP, Shames DS, et al. Vascular endothelial growth factor receptor 2 mediates macrophage infiltration into orthotopic pancreatic tumors in mice. Cancer Res. 2008; 68(11):4340-6. https://doi.org/10.1158/0008-5472.CAN-07-6705.

30. Javle M, Smyth EC, Chau I. Ramucirumab: successfully targeting angiogenesis in gastric cancer. Clin Cancer Res. 2014;20(23):5875-81. https:// doi.org/10.1158/1078-0432.CCR-14-1071.

31. Koumarianou A, Antoniou S, Kanakis G, Economopoulos N, Rontogianni D, Ntavatzikos A, et al. Combination treatment with metronomic temozolomide, bevacizumab and long-acting octreotide for malignant neuroendocrine tumours. Endocr Relat Cancer. 2012;19(1):L1-4. https://doi. org/10.1530/ERC-11-0287.

32. Mishima S, Kawazoe A, Matsumoto H, Kuboki Y, Bando H, Kojima T, et al. Efficacy and safety of ramucirumab-containing chemotherapy in patients 
with pretreated metastatic gastric neuroendocrine carcinoma. ESMO Open. 2018;3(7):e000443. https://doi.org/10.1136/esmoopen-2018-000443.

33. A'Hern RP. Sample size tables for exact single-stage phase II designs. Stat Med. 2001;20(6):859-66. https://doi.org/10.1002/sim.721.

34. Rinke AWB, Gress T, Pavel M. S2k Leitlinie Neuroendokrine Tumore. DGVS. 2018

\section{Publisher's Note}

Springer Nature remains neutral with regard to jurisdictional claims in published maps and institutional affiliations.

Ready to submit your research? Choose BMC and benefit from:

- fast, convenient online submission

- thorough peer review by experienced researchers in your field

- rapid publication on acceptance

- support for research data, including large and complex data types

- gold Open Access which fosters wider collaboration and increased citations

- maximum visibility for your research: over $100 \mathrm{M}$ website views per year

At BMC, research is always in progress.

Learn more biomedcentral.com/submissions 